\title{
Temperature dependence of the plasmonic Casimir interaction
}

\author{
F. Intravaia ${ }^{1,2}, \mathrm{H}$. Haakh ${ }^{2}$ and C. Henkel ${ }^{2}$ \\ ${ }^{1}$ Theoretical Division, MS B213, Los Alamos National Laboratory, Los Alamos, NM 87545, USA \\ ${ }^{2}$ Institut für Physik und Astronomie, Universität Potsdam, \\ Karl-Liebknecht-Straße 24/25, 14476 Potsdam, Germany
}

(Dated: 23 April 2010)

\begin{abstract}
We investigate the role of surface plasmons in the electromagnetic Casimir effect at finite temperature, including situations out of global thermal equilibrium. The free energy is calculated analytically and expanded for different regimes of distances and temperatures. Similar to the zero-temperature case, the interaction changes from attraction to repulsion with distance. Thermal effects are shown to be negligible for small plate separations and at room temperature, but become dominant and repulsive at large values of these parameters. In configurations out of global thermal equilibrium, we show that the selective excitation of surface plasmons can create a repulsive Casimir force between metal plates.
\end{abstract}

PACS numbers: 73.20.Mf - Surface plasmons, 05.30.-d - Quantum statistical mechanics, 68.35.Md Surface thermodynamics, surface energies in surfaces and interfaces

\section{INTRODUCTION}

The interaction between two parallel plates due to the zero point fluctuations of the electromagnetic field is commonly known as the Casimir effect. For metallic plates, it is well known that at short distance, the interaction can be attributed to surface plasmon modes [1, 2] that hybridize across the vacuum between the interfaces [3, 4]. Surface plasmons have been attracting much interest in the last years in connection with a broad range of topics such as near-field spectroscopy, sub-wavelength resolution [5, 6] or extraordinary optical transmission through subwavelength metallic hole arrays [7-9]. The electromagnetic field associated with these modes is evanescent. It therefore came as a surprise that they also give a large contribution at large distances, and even a repulsive one [10-12]. These papers have been restricted to the Casimir effect at zero temperature. The present paper generalizes these results by including a nonzero temperature and situations out of thermal equilibrium. One might expect that the thermal excitation of surface plasmons is irrelevant, since their typical energies are comparable to the plasma frequency of the metal, much larger than experimentally relevant thermal energies. As retardation is taken into account, however, the surface plasmon dispersion relation approaches the light cone and drops to lower frequencies. These are comparable, for two parallel plates, to the lowest cavity resonance $\sim c / L$. We find indeed a significant thermal component to the Casimir interaction between surface plasmon modes when the distance $L$ exceeds the thermal wavelength $\sim \hbar c / k_{B} T$. By selectively exciting a class of plasmonic modes, we even get an overall repulsive Casimir force.

The paper is organized as follows. We first recall the dispersion relations for coupled surface plasmon modes on two metallic plates [1] and obtain a general expression for the corresponding Casimir free energy. This is expanded asymptotically in different regimes of distance $L$ and temperature $T$ in Sec.III Sec.IV discusses the plasmonic Casimir entropy. We then compare the results to the full Casimir interaction between metal plates (Sec. $\mathbf{V}$ ), including all electromagnetic modes, consider situations in which the plasmonic modes are not at the same temperature as the rest of the system (SecVI), and conclude with a short summary.

We adopt throughout this paper the following lossless dielectric function

$$
\varepsilon(\omega)=1-\frac{\omega_{\mathrm{p}}^{2}}{\omega^{2}},
$$

where $\omega_{\mathrm{p}}$ is the plasma frequency. The Casimir energy and force (both per unit area) are normalized to the values found for perfectly reflecting mirrors [13]

$$
E_{\mathrm{C}}=-\frac{\hbar c}{4 \pi \aleph L^{3}}, \quad F_{\mathrm{C}}=-\frac{3 \hbar c}{4 \pi \aleph L^{4}},
$$

where $\aleph=180 / \pi^{3}$. An intrinsic physical length scale of the system is the plasma wavelength $\lambda_{\mathrm{p}}=2 \pi c / \omega_{\mathrm{p}}$. It is convenient to use the latter as a length scale, switching to a dimensionless plate distance $\lambda=L / \lambda_{\mathrm{p}}$. We introduce also a reduced temperature $\tau=T / T_{\mathrm{p}}=\left(2 \pi k_{B} T\right) / \hbar \omega_{\mathrm{p}}$, where $T_{\mathrm{p}}$ is the plasma temperature. This choice makes the numerical results independent of the specific material, and gives universal scaling laws. Note that $1 / \tau$ is proportional to the ratio between the thermal wavelength (a few microns at room temperature) and the plasma wavelength, and that the product $\lambda \tau=k_{B} T L /(\hbar c)$ is independent of the plasma wavelength. A parameter set used frequently in related work is the one for gold: $\hbar \omega_{\mathrm{p}}=8.96 \mathrm{eV}, \lambda_{\mathrm{p}}=136 \mathrm{~nm}$ and $T_{\mathrm{p}}=1.66 \times 10^{4} \mathrm{~K}$. Room temperature then corresponds to $\tau \approx 1.8 \times 10^{-2}$.

\section{PLASMONIC CASIMIR FREE ENERGY}

The Casimir free energy of two metallic plates is obtained by summing the free energy (per mode) over the electromagnetic modes vibrating inside the cavity [3, 13]. This expression is suitably regularized, namely by subtracting the limit of large distances between the plates. 


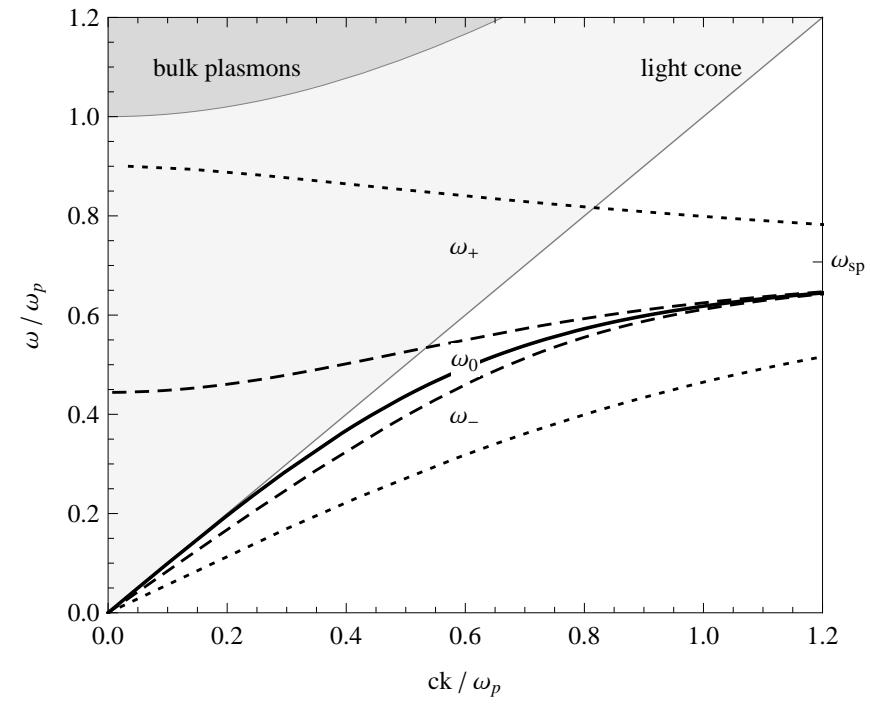

Figure 1: Dispersion relation of the surface plasmon modes at plate separation $L=c / \omega_{\mathrm{p}}$ (dotted curves) and $L=2 c / \omega_{\mathrm{p}}$ (dashed curves); $k$ is the wavevector parallel to the surface. The solid black curve $\omega_{0}(k)$ corresponds to the surface plasmon on an isolated plate $(L \rightarrow \infty)$. The branch $\omega_{+}(k)$ crosses the light cone. The asymptotic value at large $k$ is $\omega_{\mathrm{sp}}=\omega_{\mathrm{p}} / \sqrt{2}$.

Since the modes of the electromagnetic field are formally equivalent to harmonic oscillators, the free energy of a single mode of frequency $\omega$ in thermodynamical equilibrium at temperature $T$ is

$$
f(\omega)=\frac{\hbar \omega}{2}+k_{B} T \ln \left[1-e^{-\frac{\hbar \omega}{k_{B} T}}\right] .
$$

In this paper, we sum Eq. (3) over the dispersion relations for the surface plasmone modes. Isolated surfaces (at infinite distance) carry a single surface plasmon mode of frequency $\omega_{0}(k)$, illustrated in Fig. 1. If the plates are brought together, the electromagnetic fields of the modes overlap, breaking the degeneracy and splitting the dispersion relation in two branches, whose frequencies we label $\omega_{ \pm}(k)$. The modes $\omega_{-}(k)$ and $\omega_{0}(k)$ are both entirely evanescent and lie below the light cone. The mode $\omega_{+}(k)$, however, crosses the light cone and connects smoothly with the lowest propagating mode (with p-polarization) within the cavity [1]. Adding the free energies of the coupled modes and subtracting twice the corresponding values at infinite distance the integral over the dispersion relations gives the plasmonic Casimir free energy in the form (coefficients $c_{ \pm}=1, c_{0}=-2$ )

$$
\mathcal{F}(L, T)=\sum_{a= \pm, 0} c_{a} \int_{k_{a}}^{\infty} \frac{k \mathrm{~d} k}{2 \pi} f\left(\omega_{a}\right)
$$

which is convergent at large $k[10]$. The thermal part of the free energy [second term in Eq. [3] ] naturally cuts off modes above $k_{B} T / \hbar$. Different choices of the lower limits $k_{a}$, related to the subtraction procedure, are possible and have

been discussed in Refs. [11, 12, 14-16]. They are connected with the way the evanescent and propagating contributions of the mode $\omega_{+}(k)$ are split. Here we apply the convention of Refs. [11, 12] and set $k_{a}=0$ for all modes. We thus include both propagating and evanescent branches of the 'plasmonic mode' $\omega_{+}(k)$.

The calculation of the integral (4) is challenging because the surface plasmon dispersion relations $\omega_{a}(k)$ are solutions of a transcendental equation, except in the non-retarded limit $k \gg \omega_{\mathrm{p}} / c$ where $\omega_{a}^{2}(k)=\omega_{\mathrm{sp}}^{2}\left(1+a e^{-k L}\right.$ with $\omega_{\mathrm{sp}}=$ $\omega_{\mathrm{p}} / \sqrt{2}, a=0, \pm$. Progress can be made with the parametric form described in Refs. [10-12]. Adopting the notation of Ref. [11], we get the dispersion relations $\omega_{a}(k), a \in\{0, \pm\}$, from

$$
\omega_{a}^{2}(z)=\frac{c^{2}}{L^{2}} g_{a}^{2}(z), \quad k_{a}^{2}(z)=\frac{z+g_{a}^{2}(z)}{L^{2}}
$$

with the dimensionless functions

$$
g_{a}^{2}(z)=\frac{(2 \pi \lambda)^{2} \sqrt{z}}{\sqrt{z}+\sqrt{z+(2 \pi \lambda)^{2}}[\tanh (\sqrt{z} / 2)]^{a}} .
$$

(The exponents are \pm 1 for $a= \pm$.) The parameter $z$ varies from $-z_{a} \ldots \infty$ : one has $z_{0}=z_{-}=0$, while the number $z_{+}$, plotted in Fig.2] as a function of $\lambda$, is the solution[11] of the transcendental equation $\sqrt{z_{+}}=2 \pi \lambda \cos \left(\sqrt{z_{+}} / 2\right)$. The propagating branch of the mode $\omega_{+}(k)$ corresponds to the interval $z \in\left[-z_{+}, 0\right]$; evanescent modes (below the light cone) have $z>0$. Changing the integration variable in Eq. (4) from $k$ to $z$, the plasmonic Casimir free energy is given by

$$
\mathcal{F}=\frac{\hbar c}{8 \pi L^{3}} \sum_{a= \pm, 0} c_{a}\left[\int_{-z_{a}}^{\infty}\left(g_{a}(z)+2 \lambda \tau \ln \left[1-e^{-\frac{g_{a}(z)}{\lambda \tau}}\right]\right) \mathrm{d} z+2 \int_{g_{a}\left(-z_{a}\right)}^{g_{a}(\infty)}\left(g_{a}^{2}+2 g_{a} \lambda \tau \ln \left[1-e^{-\frac{g_{a}}{\lambda \tau}}\right]\right) \mathrm{d} g_{a}\right]
$$

For all plasmonic modes, the second integral has a finite up-

per limit $g_{a}(\infty)=\sqrt{2} \pi \lambda=\omega_{\mathrm{sp}} L / c$ that coincides with 
the non-retarded surface plasmon frequency; their contributions cancel in the sum over $a$. The lower boundaries are $g_{0}\left(-z_{0}\right)=g_{-}\left(-z_{-}\right)=0$, and $g_{+}\left(-z_{+}\right)=\sqrt{z_{+}}$. The first integral in Eq. (7) can only be evaluated approximately (see Sec. III), but a closed form can be given for the second one.

In the following, we scale the plasmonic free energy to the zero-temperature Casimir value, Eq. (2),

$$
\mathcal{F}(L, T)=E_{\mathrm{C}}(L) \varphi(\lambda, \tau)
$$

and split the correction factor in two terms

$$
\varphi(\lambda, \tau)=\eta(\lambda)+\vartheta(\lambda, \tau)
$$

where the first is the plasmonic Casimir energy at zero temperature [11, 12]:

$$
\eta(\lambda)=-\frac{\aleph}{2} \sum_{a} c_{a} \int_{-z_{a}}^{\infty} g_{a}(z) \mathrm{d} z+\frac{\aleph}{3} z_{+}^{3 / 2} .
$$

The second term in Eq. 9) gives the temperature-dependent part for which Eq.(7) gives

$$
\begin{aligned}
\vartheta(\lambda, \tau)= & -\aleph \lambda \tau \sum_{a} c_{a} \int_{-z_{a}}^{\infty} \ln \left[1-e^{-\frac{g_{a}(z)}{\lambda \tau}}\right] \mathrm{d} z \\
& -2 \aleph(\lambda \tau)^{3} \mathcal{L}\left(\frac{\sqrt{z_{+}}}{\lambda \tau}\right)
\end{aligned}
$$

where the following combination of polylogarithmic functions appears

$$
\mathcal{L}(x)=\zeta(3)-\operatorname{Li}_{3}\left(e^{-x}\right)-x \operatorname{Li}_{2}\left(e^{-x}\right)
$$

with $\operatorname{Li}_{n}(x)=\sum_{k=1}^{\infty} x^{k} / k^{n}$. We note that $\mathcal{L}(x) \sim \frac{1}{4} x^{2}(1-$ $2 \log x)$ for small $x$, and $\mathcal{L}(x) \rightarrow \zeta(3)$ exponentially fast for large $x$. Eq. 10b does not depend only on the product $\lambda \tau$ because the material-dependent parameter $\lambda$ enters via the lower limit $z_{+}$and the functions $g_{a}(z)$ [Eq. [6] ].

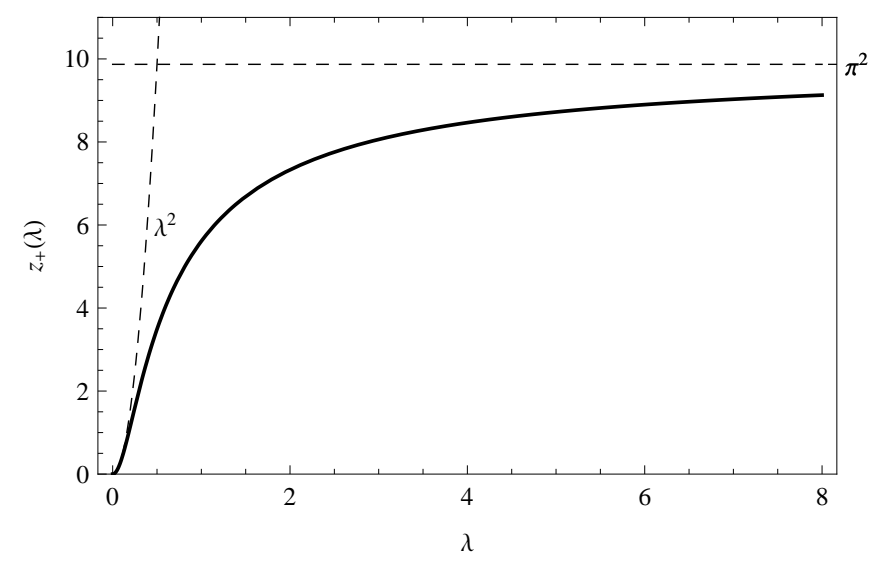

Figure 2: $z_{+}(\lambda)$ vs. the plate separation $\lambda$ and its asymptotes at small and large distances. The limiting cases are $z_{+} \approx(2 \pi \lambda)^{2}$ and $\approx \pi^{2}$ for $\lambda \rightarrow 0$ and $\rightarrow \infty$, respectively.

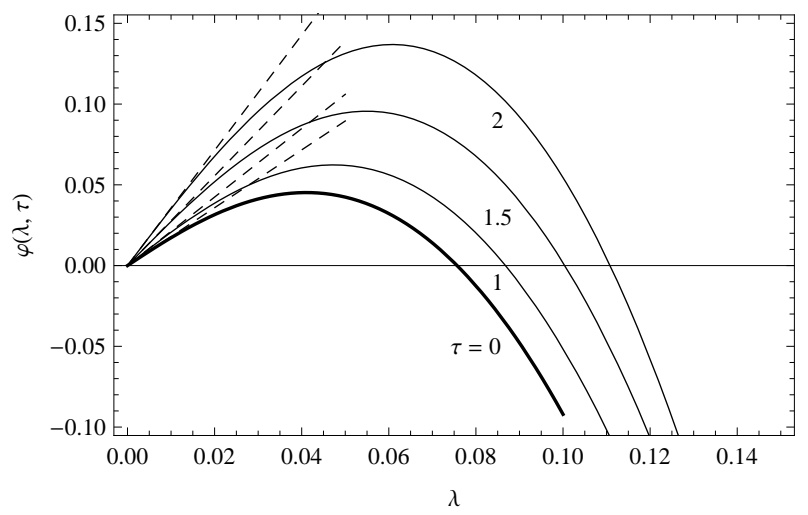

Figure 3: Plasmonic contribution to the Casimir free energy vs. distance at different temperatures, normalized to the perfect mirror case at $T=0$ (energy correction factor $\varphi(\lambda, \tau)$ in Eq. 8 ). Solid curves: numerical evaluation of Eqs. 10a 10b for different temperatures; dashed curves: short-distance limit Eqs. 15, 16. Distance and temperature are scaled to the plasma wavelength $2 \pi c / \omega_{\mathrm{p}}$ and temperature $T_{\mathrm{p}}=\hbar \omega_{\mathrm{p}} / k_{B}$, respectively. Negative values correspond to a repulsive interaction energy.

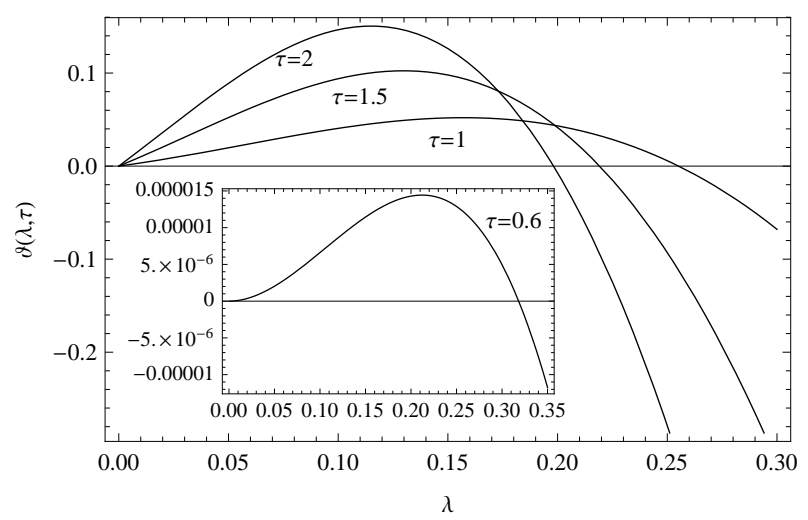

Figure 4: Thermal correction to the plasmonic free energy at short distance for different temperatures (energy correction factor $\vartheta(\lambda, \tau)$ in Eq.91). The behavior qualitatively differs from the complete free energy (Fig.3), as a quadratic (rather than linear) distance dependence emerges at low temperatures, cf. Eq. 16.

The Casimir free energy is shown in Figs. 3, 4, 7 and 8 for different distance ranges. Qualitatively, a nonzero temperature does not modify the behavior of the plasmonic contribution - we still get a sign change at a distance of order $\lambda_{\mathrm{p}} / 2 \pi$, with the interaction becoming repulsive at large distances. We plot in Fig.5 the inversion distance where the Casimir pressure, $-\partial \mathcal{F} / \partial L$, changes sign: a weak increase is found as the temperature is raised. Much larger changes will be found in $\mathrm{Sec}$ VI where configurations out of thermal equilibrium are discussed.

In the following, we analyze the thermal correction $\vartheta(\lambda, \tau)$ in different regimes of distance and temperature. The zerotemperature Casimir energy $\eta(\lambda)$ depends only on one physical scale provided by the plasma wavelength and leads to two regimes $\lambda \ll 1$ and $\lambda \gg 1$. For $\vartheta(\lambda, \tau)$ we discuss three 


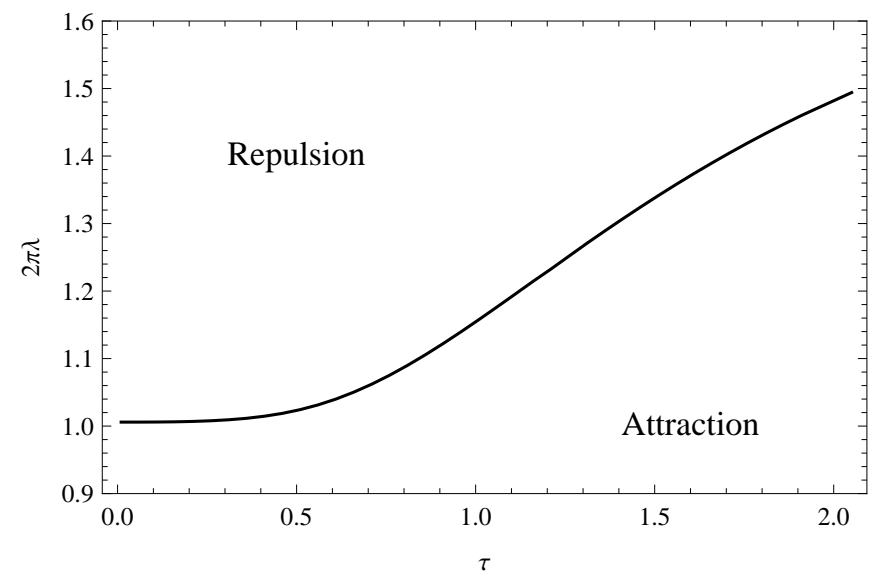

Figure 5: Repulsive and attractive regimes of the plasmonic Casimir pressure in the $(\lambda, \tau)$-plane. A nonzero temperature slightly increases the distance for which the plasmonic contribution to the Casimir force becomes repulsive.

regimes. In all situations of practical interest, it is safe to assume $\tau \ll 1$ for the scaled temperature and we can distinguish between: short distances $\lambda \ll 1$, intermediate distances, $1 \ll \lambda \ll 1 / \tau$, and large distances (beyond the thermal wavelength) $1 / \tau \ll \lambda$.

\section{ASYMPTOTIC EXPRESSIONS}

The calculation of asymptotic expressions requires some care already at zero temperature as was shown in Ref. [12]. When performing approximations on the integrals in Eqs. (10a) and 10b one must bear in mind that the functions $g_{a}(z)$ cover a wide range of values, from very small to large, depending on $z$ and $\lambda$. Their characteristic scale in the variable $z$ is given by the distance parameter $\lambda$. For $-z_{a}<z \ll(2 \pi \lambda)^{2}$ we may use $(a=0, \pm)$

$$
g_{a}(z) \approx \sqrt{2 \pi \lambda \sqrt{z}\left[\operatorname{coth}\left(\frac{\sqrt{z}}{2}\right)\right]^{a}}
$$

while for $z \gg(2 \pi \lambda)^{2}$ the (non-retarded) approximations

$$
g_{a}(z) \approx \frac{2 \pi \lambda}{\sqrt{2}} \sqrt{1+a e^{-\sqrt{z}}}
$$

hold. It is therefore convenient to split the integration range in Eqs. 10a and 10b as follows

$$
\int_{-z_{a}}^{\infty} \mathrm{d} z=\int_{-z_{a}}^{0} \mathrm{~d} z+\int_{0}^{(2 \pi \lambda)^{2}} \mathrm{~d} z+\int_{(2 \pi \lambda)^{2}}^{\infty} \mathrm{d} z .
$$

The first integral concerns only the mode $\omega_{+}(k)$ because $z_{-}, z_{0}=0$. We can use Eq. (12) in the first two integrals and Eq. (13) in the third. Depending on distance and temperature and on the desired accuracy, these have to be compared with the integrated terms in Eq. (10) (proportional to $z_{+}^{3 / 2}$ or $\left.\mathcal{L}\left(\sqrt{z_{+}} / \lambda \tau\right)\right)$.

\section{A. Short distance}

At short distance the zero-temperature energy correction was already analyzed in Ref. [12]. It turns out that it is dominated by large values of $z$ (third integral of Eq. (14)). At the leading order, we get

$$
\eta(\lambda) \stackrel{\lambda \ll 1}{\longrightarrow} 1.790 \lambda .
$$

Higher order terms take the form $\lambda^{3}(a+b \log \lambda)$ with numerical coefficients $a$ and $b$ given in the same reference.

Considering the thermal correction for $\lambda \ll 1$, the thermal scale becomes important, too. For realistic temperatures, we also have $\lambda \tau \ll 1$, and the main contribution arises from the second and the third integral in Eq. (14). Indeed, it can be shown that the first integral and the polylogarithmic term (involving $\mathcal{L}$ ) are beyond the order $\mathcal{O}\left(\lambda^{2}\right)$. In addition, the main contribution to the second integral arises from the mode $\omega_{-}(k)$. This is not surprising since the thermal correction selects frequencies $\omega_{a}(k) \lesssim T$ and the mode $\omega_{-}(k)$ is the one that vanishes most quickly as $k \rightarrow 0$. The corresponding exponent in $k$ determines the power law in $\tau$, as we discuss at the end of this section. The opposite case $\lambda \tau \gg 1$ is physically irrelevant at short distances, because one would need $\tau \gg 1$. Mathematically, one finds a divergence from the term proportional to $\mathcal{L}$ in Eq. $10 \mathrm{~b}$ that is exactly balanced by the first integral in Eq. (14). It follows that the asymptotic form given in Eq. 16 remains valid.

All told, up to the second order in $\lambda$ we find

$$
\vartheta(\lambda, \tau) \stackrel{\lambda \ll 1}{\longrightarrow} \aleph \lambda \tau\left[2 \frac{\lambda \tau^{2}}{\pi} \mathcal{L}\left(2 \pi \sqrt{\pi \lambda / \tau^{2}}\right)+\beta(\tau)\right]
$$

where the function $\mathcal{L}(x)$ defined in Eq. (11) appears with a different argument, and where the temperature-dependent function $\beta(\tau)$ is

$$
\begin{aligned}
\beta(\tau)= & \int_{0}^{\infty} \ln \left[\frac{1-e^{-\pi \sqrt{2} / \tau}}{1-e^{-\pi \sqrt{2\left(1+e^{-\sqrt{z}}\right)} / \tau}}\right] \mathrm{d} z \\
& +\int_{0}^{\infty} \ln \left[\frac{1-e^{-\pi \sqrt{2} / \tau}}{1-e^{-\pi \sqrt{2\left(1-e^{-\sqrt{z}}\right.} / \tau}}\right] \mathrm{d} z
\end{aligned}
$$

This is plotted in Fig.6 together with its asymptotes in the limits of high and low temperatures,

$$
\beta(\tau) \stackrel{\tau \ll 1}{\longrightarrow} 6 \zeta(5)\left(\frac{\tau}{\pi}\right)^{4}, \quad \beta(\tau) \stackrel{\tau \rightarrow \infty}{\longrightarrow} \frac{\zeta(3)}{4} .
$$

We observe the emergence of the characteristic ratio $\lambda / \tau^{2}$ that determines which of the two terms in Eq. (16) dominates. This illustrates that the limits $\lambda \rightarrow 0$ and $\tau \rightarrow 0$ do not commute for the temperature-dependent Casimir energy. If $\lambda \ll \tau^{2}$ (extremely short distances or high temperatures, main plot of Fig.4), the function $\beta(\tau)$ governs the thermal correction $\vartheta$ which scales as $\vartheta \sim \lambda \tau^{5}$ if $\lambda \ll \tau^{2} \ll 1$. We recover here the same linear distance dependence as at zero temperature, Eq. [15). The opposite regime $\tau^{2} \ll \lambda \ll 1$ emerges 


\section{B. Intermediate distance}

at low temperatures, where the term involving $\mathcal{L}$ in Eq. (16) dominates: we get a behavior $\vartheta \sim \lambda^{2} \tau^{3}$ (inset of Fig. (4). This crossover from a quadratic to a linear scaling with distance can be seen in Fig. 4

This discussion also illustrates the failure of the nonretarded approximation. This leads to surface plasmon dispersion relations $\omega_{ \pm}^{2}(k)=\omega_{\mathrm{sp}}^{2}\left(1 \pm e^{-k L}\right)$ and $\omega_{0}=\omega_{\mathrm{sp}}$, and extrapolates a free energy $\vartheta \sim \tau^{5}$ down to low temperatures, while the correct power is $\tau^{3}$. This is of course crucial for the low-temperature expansion of a thermodynamic quantity like the entropy (see Section IV.

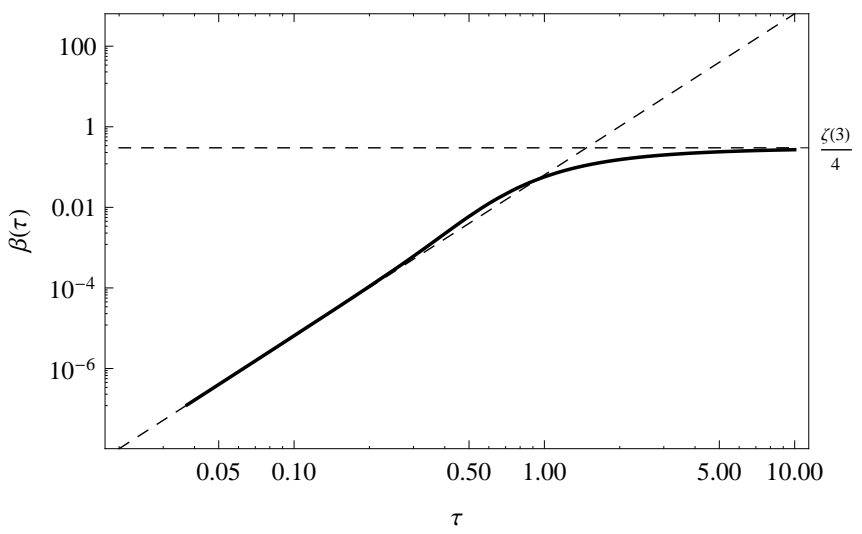

Figure 6: The function $\beta(\tau)$ vs. $\tau$ and its asymptotes 18 at low and high temperatures.

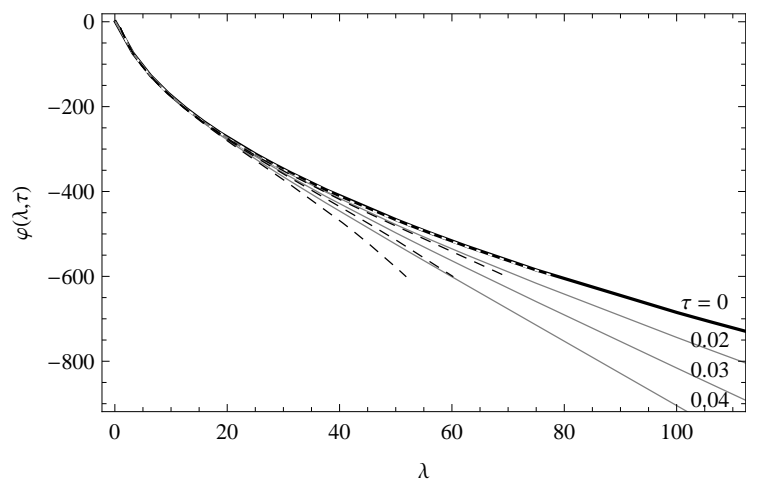

Figure 7: Thermal plasmonic Casimir free energy (reduction factor $\varphi(\lambda, \tau)$ in Eq. 8) at intermediate distances and different temperatures. Exact numerical calculation (solid curves) and the approximation (19) at zero temperature (short-dashed white) and sum of Eqs. 19] and 20) in the intermediate regime $\lambda \tau \ll 1$ (long-dashed) respectively .
Parameters for typical experiments are $\tau \approx 10^{-2}, \lambda \approx$ $1 \ldots 10^{2}$, way beyond short distances. They lie inside an intermediate regime $1 \ll \lambda \ll 1 / \tau$, where the plate distance is between the plasma and the thermal wavelength. Here, the thermal correction to the free energy is still small compared to zero temperature, as for short distances.

In the scaled Casimir energy $\eta(\lambda, \tau)$, the main contribution to the integral 10a arises for $z \sim 1$ and we have to consider the first two integrals in Eq. (14), where, approximately, $z_{+} \approx \pi^{2}$. The energy correction factor at zero temperature then becomes [12]

$$
\eta(\lambda) \stackrel{\lambda \gg 1}{\longrightarrow}-74.57 \sqrt{\lambda}+60 .
$$

the offset arising from the second term in Eq.10a.

For the thermal correction, we find the leading order from the polylogarithmic $\mathcal{L}$ in Eq. $10 \mathrm{~b}$. The $z$-integral gives a contribution which is dominated by the interval $z=$ $-z_{+} \ldots(2 \pi \lambda)^{2}$. Combining the two,

$$
\vartheta(\lambda, \tau) \approx-2 \aleph(\lambda \tau)^{3} \zeta(3)\left(1-\frac{1}{\lambda \pi}\right)
$$

This gives a small correction that scales as $\tau^{3}$. Both Eqs. 19] and (20) are plotted in Fig.77 illustrating the weak impact of temperature. It is interesting to note that in this range of distances, the thermal plasmonic contribution is opposite in sign to the free energy of the full electromagnetic Casimir effect (see Sec.IV), and increases the plasmonic repulsion.

\section{Large distance}

Let us finally consider the regime $\lambda \gg 1 / \tau \gg 1$, corresponding to a plate separation larger than both the plasma and the thermal wavelength. The zero-temperature contribution can still be approximated by Eq. [19, , but now the thermal contribution dominates the free Casimir energy. The asymptotic behavior of the integrals in Eq. $10 \mathrm{~b}$ is obtained by expanding the logarithms for small $g_{a}(z) /(\lambda \tau)$, since the functions $g_{a}(z)$ are bounded:

$$
-\lambda \tau \ln \left[1-e^{-\frac{g_{a}(z)}{\lambda \tau}}\right] \approx-\lambda \tau \ln \left[\frac{g_{a}(z)}{\lambda \tau}\right]+\frac{g_{a}(z)}{2}+\cdots
$$

Using this expansion under the integral in Eq. 10b, we note that the second term balances exactly with the zerotemperature contribution from Eq. 10a). As for the first term, we perform the $z$-integration by splitting the integration range as in Eq. (14). It is easy to see that in the second interval, the sum over the mode branches gives zero. The leading contribution now comes from negative $z$, while the third interval in Eq. (14) gives an exponentially small contribution $\sim e^{-4 \pi \lambda}$. The polylogarithmic term $\mathcal{L}$ can be expanded for small argu- 


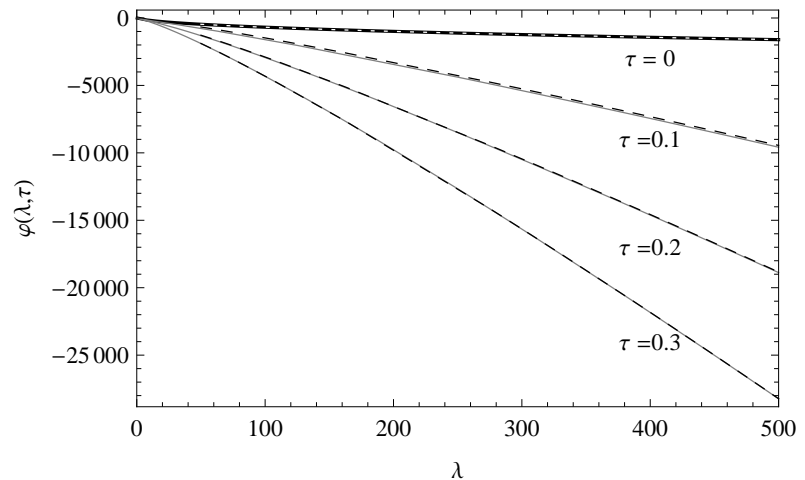

Figure 8: Plasmonic Casimir free energy (correction factor $\varphi(\lambda, \tau)$ ) at large distances and different temperatures. Numerical calculation (solid curves) and large-distance approximations 19 ) at zero temperature (white dotted line) and 22 at non-zero temperature (dashed curves).

ment, which gives eventually

$$
\begin{aligned}
\varphi(\lambda, \tau) \underset{\lambda \gg 1}{\stackrel{\lambda \tau \gg 1}{\longrightarrow}} & -\frac{\aleph \lambda \tau}{2} \int_{-\pi^{2}}^{0} \ln \left[\frac{2 \pi}{\lambda \tau^{2}} \sqrt{z} \operatorname{coth}\left(\frac{\sqrt{z}}{2}\right)\right] \mathrm{d} z \\
& -2 \aleph(\lambda \tau)^{3} \mathcal{L}\left(\frac{\pi}{\tau \lambda}\right) \\
& \approx-\frac{\aleph \pi^{2} \lambda \tau}{2}\left(\ln (2 \lambda)-\frac{7 \zeta(3)}{\pi^{2}}+\frac{1}{2}\right)
\end{aligned}
$$

where the dependence on $\ln \tau$ cancels to leading order. The validity range of this asymptotic formula is illustrated in Fig. 8 (dashed lines) where the full free energy is plotted at large distances.

Summarizing this section, we have generalized a result known from the zero-temperature case [11, 12] to $T>0$ : only the branch of the plasmonic mode $\omega_{+}(k)$ that crosses into the propagating sector contributes to the (repulsive) plasmonic Casimir interaction at large distances in a significant way.

\section{PLASMONIC CASIMIR ENTROPY}

The plasmonic Casimir entropy can be derived from the plasmonic Casimir free energy, Eq. (7), by differentiation with respect to $T$,

$$
S(L, T)=-\frac{\partial \mathcal{F}}{\partial T}=S_{\mathrm{C}}(L) \sigma(\lambda, \tau) .
$$

A convenient scale is given by the Casimir entropy at high temperatures between two perfect reflectors (this includes two transverse photon polarizations)

$$
S_{\mathrm{C}}(L)=\frac{\zeta(3)}{8 \pi} \frac{k_{B}}{L^{2}} .
$$

The scaled entropy is connected to the dimensionless thermal correction $\vartheta(\lambda, \tau)$ by a derivative (mind that $E_{\mathrm{C}}<0$ )

$$
\sigma(\lambda, \tau)=\frac{2}{\zeta(3) \aleph \lambda} \frac{\partial}{\partial \tau} \vartheta(\lambda, \tau) .
$$

We recall the result for perfect reflectors where the entropy depends only on the product $\lambda \tau$ [17]

$$
\sigma_{\mathrm{C}}(\lambda, \tau)= \begin{cases}12(\lambda \tau)^{2}, & \lambda \tau \ll 1 \\ 1, & \lambda \tau \gg 1\end{cases}
$$

The Casimir entropy due to surface plasmons can be represented as the integral

$$
\sigma(\lambda, \tau)=-\frac{4}{\zeta(3)}\left(\sum_{a} \frac{c_{a}}{2} \int_{-z_{a}}^{\infty}\left(\ln \left[1-e^{-\frac{g_{a}(z)}{\lambda \tau}}\right]-\bar{n}_{a}(z) \frac{g_{a}(z)}{\lambda \tau}\right) \mathrm{d} z+3(\lambda \tau)^{2} \mathcal{L}\left(\frac{\sqrt{z_{+}}}{\lambda \tau}\right)+z_{+} \ln \left[1-e^{-\frac{\sqrt{z^{+}}}{\lambda \tau}}\right]\right)
$$

where $\bar{n}_{a}(z)=\left[\exp \left(g_{a}(z) / \lambda \tau\right)-1\right]^{-1}$ is the Bose-Einstein mean photon number. Figs.9, 10 show the temperature dependence of $\sigma(\lambda, \tau)$ for several distances $\lambda$, small and large. The strong qualitative differences between these cases could be anticipated from the free energy of Fig.4 at short distances, temperature makes increase $\vartheta(\lambda, \tau)$ towards positive values, leading to a positive $\sigma$ from Eq. [25], while the trend is reversed at larger distances.

Note from Figs 9 and 10 that the plasmonic Casimir entropy fulfills Nernst's heat theorem $(\sigma \rightarrow 0$ as $T \rightarrow 0)$ at all distances. Though the same is known for the entropy of the complete plasma model [18] including photonic modes, the result is not trivial because surface plasmons are only a subsystem of the two-plate system.

As before one can distinguish three characteristic distance regimes for the plasmonic Casimir entropy. The expression for $\lambda \ll 1$ can be easily obtained from the approximation (16) to $\vartheta(\lambda, \tau)$. At low temperatures (regime $\tau^{2} \ll \lambda \ll 1$ ), we must include a subleading term in the small- $\lambda$ expansion to get the right prefactor of the temperature power law. This is done by adding to Eq. (16) the polylogarithmic term with $\mathcal{L}$ of 


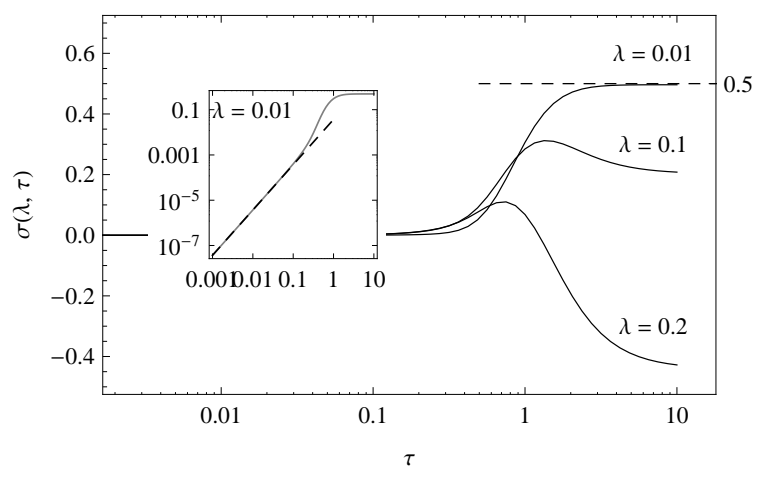

Figure 9: Temperature dependence of the Casimir entropy from plasmonic modes [correction factor $\sigma(\lambda, \tau)$ relative to perfectly conducting mirrors, Eq. [23] ] for short distances and high-temperature limit (28) (dashed). Inset (double logarithmic scale): low-temperature behavior at short distance and its asymptote (dashed) $\sigma \sim \tau^{2}$ from Eq. 27.

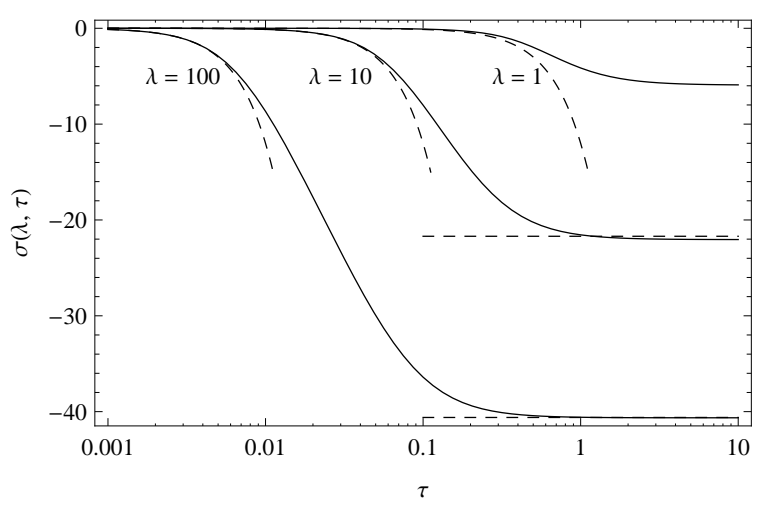

Figure 10: Plasmonic Casimir entropy in the scaled form $\sigma(\lambda, \tau)$ [Eq. [23] ], vs. temperature for intermediate and large distances (solid lines). Dashed: low- and high-temperature asymptotes 29], 30].

Eq. (10b) that becomes $-2 \aleph(\lambda \tau)^{3} \zeta(3)$. Differentiation leads to

$$
\sigma(\tau, \lambda) \underset{\tau \ll 1}{\stackrel{\lambda \ll 1}{\longrightarrow}} \sigma_{\mathrm{C}}(\lambda, \tau)\left[\frac{1}{\pi \lambda}+\frac{5}{\pi^{2}} \frac{\zeta(5)}{\zeta(3)}\left(\frac{\tau}{\pi \lambda}\right)^{2}-1\right] .
$$

The entropy approaches zero quadratically as $\tau \rightarrow 0$, as for perfect reflectors [Eq. (26)], but the prefactor is larger by a factor $1 /(\pi \lambda)$. The good agreement with the exact result can be seen in the inset of Fig. 9

At high temperature, $\sigma(\lambda, \tau)$ becomes a constant that coincides at short distances with the perfect reflector limit for one polarization

$$
\sigma(\lambda, \tau) \underset{\tau \gg 1}{\stackrel{\lambda \ll 1}{\longrightarrow}} \frac{1}{2} .
$$

This can be seen from Eq. (16) taking into account the function $\beta(\tau)$.

Intermediate $(1 \ll \lambda \ll 1 / \tau)$ and large $(1,1 / \tau \ll \lambda)$ distances can be treated with Eqs. (20), (22) that give, respec- tively,

$$
\begin{aligned}
& \sigma(\lambda, \tau) \underset{\lambda \gg 1}{\stackrel{\lambda \tau \ll 1}{\longrightarrow}} \sigma_{\mathrm{C}}(\lambda, \tau)\left(-1+\frac{1}{\lambda \pi}\right) . \\
& \sigma(\lambda, \tau) \underset{\lambda \gg 1}{\stackrel{\lambda \tau \gg 1}{\longrightarrow}}-\frac{\pi^{2}}{\zeta(3)}\left(\ln (2 \lambda)-\frac{7 \zeta(3)}{\pi^{2}}+\frac{1}{2}\right) .
\end{aligned}
$$

The validity range of these formulas can be seen from Fig.10 (dashed lines). Note that for intermediate and large distances, the entropy approaches zero from below as $\tau \rightarrow 0$.

\section{PLASMONIC VS. PHOTONIC MODES}

We now compare the plasmonic Casimir interaction to the full system where all electromagnetic modes of the cavity are included. The knowledge of the surface plasmon contribution provides some physical interpretation for the complete system. For example, it is well known [3, 4] that the full Casimir interaction (zero temperature) at small plate separations is well described by taking only the electrostatic interaction between surface plasmons. We now show that this remains valid at nonzero temperature. The fundamental reason is that at short distances $(\lambda \ll 1)$, the lowest cavity modes (above the plasmonic $\omega_{-}+(k)$ ) have a characteristic frequency $2 \pi c / L$ that already falls in the transparency band of the mirrors $\left(\omega>\omega_{\mathrm{p}}\right)$.

The full Casimir free energy $\mathcal{F}_{\text {Lif }}=\varphi_{\text {Lif }}(\lambda, \tau) E_{\mathrm{C}}$ can be obtained from the Lifshitz formula [18, 19]. In our scaled units,

$$
\begin{aligned}
\varphi_{\mathrm{Lif}}(\lambda, \tau) & =-2 \aleph \lambda \tau \sum_{p} \sum_{n=0}^{\infty} \Gamma_{p}(2 \pi n \lambda \tau), \\
\Gamma_{p}(X) & =\int_{X}^{\infty} \mathrm{d} \kappa \kappa \log \left[1-r_{p}^{2}(\mathrm{i} X, \kappa) e^{-2 \kappa}\right] .
\end{aligned}
$$

The index $p \in\{\mathrm{TE}, \mathrm{TM}\}$ denotes the polarization. The numbers $X_{n}=2 \pi n \lambda \tau$ are scaled Matsubara frequencies. The Fresnel reflection coefficients in terms of the variables $\kappa$ and $X$ are

$$
r_{\mathrm{TE}}(\mathrm{i} X, \kappa)=\frac{\kappa-\kappa_{m}}{\kappa+\kappa_{m}}, \quad r_{\mathrm{TM}}(\mathrm{i} X, \kappa)=\frac{\tilde{\varepsilon}(\mathrm{i} X) \kappa-\kappa_{m}}{\tilde{\varepsilon}(\mathrm{i} X) \kappa+\kappa_{m}}
$$

with $\kappa_{m}=\sqrt{\kappa^{2}+(2 \pi \lambda)^{2}}$ and the dielectric function of the plasma model [cf. Eq. [1]] $\tilde{\varepsilon}(\mathrm{i} X)=1+(2 \pi \lambda / X)^{2}$.

Fig.11 shows the scaled free energies for both the full Casimir interaction (dashed) and the plasmonic contribution alone (solid). A good agreement is visible at short distances even at nonzero temperature. In this regime, we can, therefore, obtain detailed information on the thermodynamics of the Casimir effect by just considering the plasmonic contribution which can be worked out in analytic form quite easily. For example, we can immediately conclude that the (full) Casimir entropy at $\lambda \ll 1$ is given by Eq. (27) at low temperatures $(\tau \ll 1)$ and by Eq. 28 for $\tau \gg 1$. 


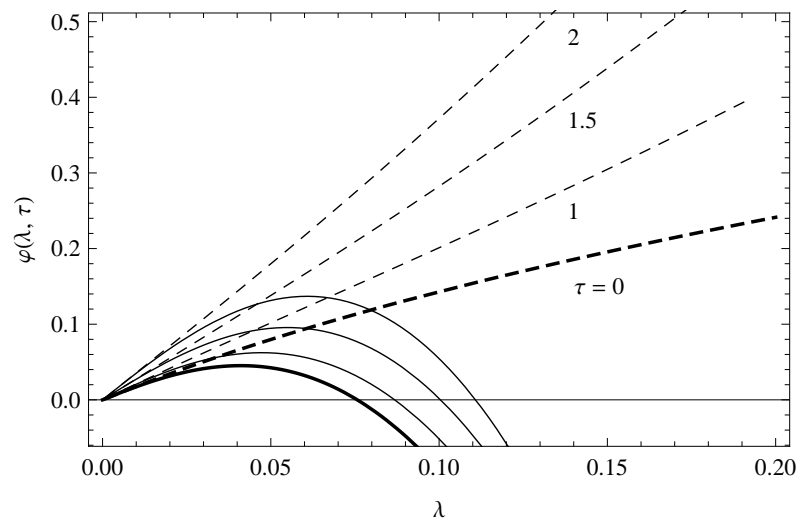

Figure 11: Casimir free interaction energy including all modes (Lifshitz theory with the plasma model, dashed lines), compared to its plasmonic counterpart (solid). All energies are expressed relative via the correction factor $\varphi(\lambda, \tau)$.

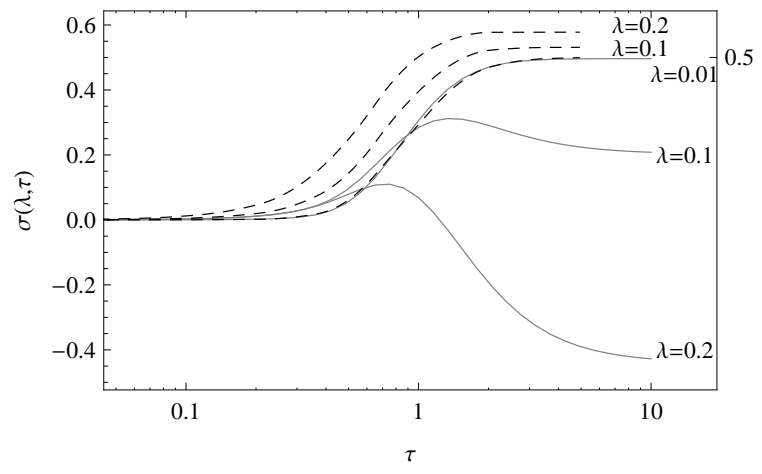

Figure 12: Casimir entropy vs. temperature for all modes (plasma model, dashed), compared to the contribution of plasmonic modes only (solid). Entropies expressed via the correction factor $\sigma(\lambda, \tau)$.

With respect to the Casimir entropy, Figs.11 and 12 illustrate that the Lifshitz expression deviates significantly from the plasmonic contribution when $\lambda \gtrsim 0.1$. It is obvious that propagating (photonic) modes then become relevant. In Appendix $₫$ we calculate their contribution at low temperatures for the plasma model:

$$
\sigma_{\mathrm{ph}}(\lambda, \tau) \underset{\tau \ll 1}{\stackrel{\lambda \tau \ll 1}{\longrightarrow}} \sigma_{\mathrm{C}}(\lambda, \tau)\left[2-\frac{8 \pi^{2} \tau}{45 \zeta(3)} \frac{2+\pi \lambda}{3}\right]
$$

where the first term is twice the value obtained for perfect mirrors. This is precisely compensated by the plasmonic contribution. Indeed, for intermediate distances $1 \ll \lambda \ll 1 / \tau$, both Eqs. (29) and (34) are valid, and their sum reproduces the entropy of the full Casimir effect calculated in Refs. [18, 2022]. Evaluating Eq. (19) of Ref. [21] in the regime of intermediate distances, we have

$$
\sigma_{\mathrm{Lif}}(\lambda, \tau) \underset{\lambda \gg 1}{\stackrel{\lambda \tau \ll 1}{\longrightarrow}} \sigma_{\mathrm{C}}(\lambda, \tau)\left[1+\frac{1}{\pi \lambda}-\frac{8 \pi^{2} \tau}{45 \zeta(3)} \frac{\pi \lambda+2}{3}\right]
$$

\section{BEYOND THERMAL EQUILIBRIUM}

Until now, we have assumed both metallic slabs to be at the same temperature $T$. The previous results enable us to deal in a simple way with a more general situation, too, where each of the (otherwise identical) slabs is described by a local temperature $T_{1}$ and $T_{2}$. The general theory in this case was investigated in Refs. [23, 24]: the non-equilibrium Casimir interaction for a symmetric cavity is obtained by simply averaging over the equilibrium free energies of the two mirrors

$$
\mathcal{F}^{\text {neq }}\left(L, T_{2}, T_{1}\right)=\frac{1}{2}\left[\mathcal{F}^{\mathrm{eq}}\left(L, T_{2}\right)+\mathcal{F}^{\mathrm{eq}}\left(L, T_{1}\right)\right] .
$$

(In Ref. [24], this result was derived for the pressure, but the same reasoning can be applied for the free energy.) Combining this formalism with the results from the present paper, it is straightforward to calculate the plasmonic contribution to the non-equilibrium Casimir free energy. Using the split (9) of the plasmonic free energy into a zero-temperature and a thermal part, Eq. (36) gives for two slabs at different temperatures

$$
\varphi^{\text {neq }}\left(\lambda, \tau_{2}, \tau_{1}\right)=\eta(\lambda)+\frac{1}{2}\left[\vartheta\left(\lambda, \tau_{2}\right)+\vartheta\left(\lambda, \tau_{1}\right)\right] .
$$

From the results given above, we conclude that qualitatively, the behavior of $\varphi^{\text {neq }}\left(\lambda, \tau_{2}, \tau_{1}\right)$ is similar to the equilibrium configuration, including a change in sign of the force with the distance. This is also confirmed by the asymptotic expressions for long/short distance and low/high temperature that can be easily extracted from the above results. The total Casimir force between identical plates, however, is always attractive, as is known from Refs. [23, 24] for all temperatures.

Let us now consider a slightly different non-equilibrium scenario where temperature is still raised locally (in one plate), but only for a subclass of modes. If it were possible to increase the mean excitation of the plasmonic modes on one plate, above the equilibrium level of the propagating (photonic) modes, the total Casimir free energy would read

$$
\varphi_{\mathrm{Lif}}^{\mathrm{neq}}\left(\lambda, \tau_{2}, \tau_{1}\right)=\varphi_{\mathrm{Lif}}\left(\lambda, \tau_{1}\right)+\frac{1}{2}\left[\vartheta\left(\lambda, \tau_{2}\right)-\vartheta\left(\lambda, \tau_{1}\right)\right],
$$

where the first term is the total Casimir free energy at equilibrium. The set of curves b) in Fig.13 illustrates that this scenario can create a regime where the total Casimir force becomes repulsive, and this over a fairly large range of distances. We plot the Casimir pressure (non-equilibrium force per unit area) when the photonic modes are either at the scaled temperature $\tau_{1}$ (zero or room temperature), and the plasmonic modes on plate 2 at $\tau_{2}>\tau_{1}$. It appears that this setting breaks the delicate balance between photonic and plasmonic modes we found in Sec. V $\mathrm{V}$ A similar interpretation has been put forward in Ref. [25] for the change in distance dependence of the atom-surface interaction out of equilibrium. The two values for $\tau_{1}$ give close results because in the intermediate distance range, the effect of the temperature is still moderate for the equilibrium case. As could be expected, the inversion distance increases and the maximal repulsion becomes weaker as $\tau_{1}$ increases towards $\tau_{2}$. 


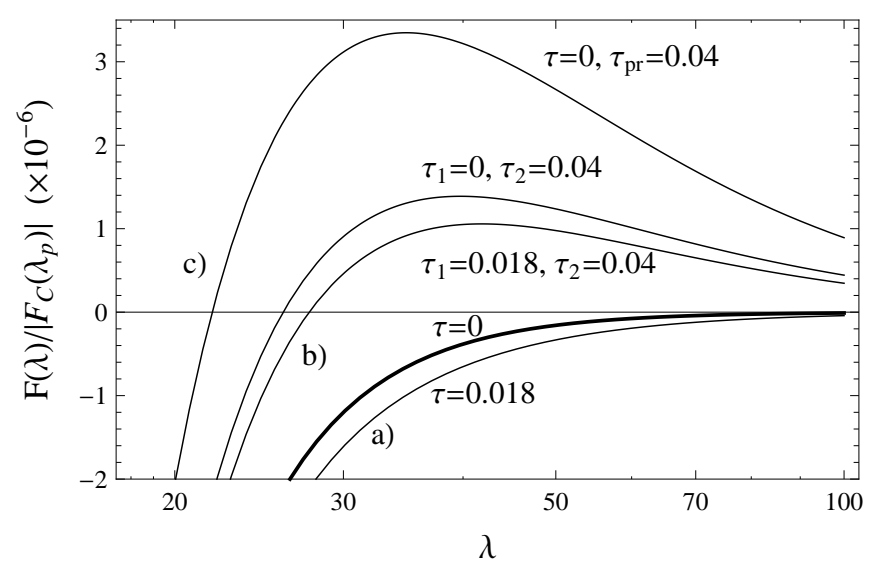

Figure 13: Total Casimir force (per unit area) in thermal equilibrium (thick line) and in different non-equilibrium scenarios. The force is normalized to $10^{-6}\left|F_{\mathrm{C}}\left(\lambda_{\mathrm{p}}\right)\right|$, approximately $3.65 \mu \mathrm{Pa}$ for gold $\left(\lambda_{\mathrm{p}}=136 \mathrm{~nm}\right)$. In these units, $T=300 \mathrm{~K}(665 \mathrm{~K})$ corresponds to $\tau \approx 0.018$ (0.04), respectively.

a) Total equilibrium at temperature $\tau$ : attractive pressure at all distances. b) Surface plasmon modes of one plate out of equilibrium at temperature $\tau_{2}$, all other modes at temperature $\tau_{1}$. c) All modes at $\tau$, except for the propagating branch of the plasmonic mode $\left(\omega_{+}(k)\right)$ which is at temperature $\tau_{\mathrm{pr}}$. The sign change to repulsion (positive pressure) would occur for gold at distances between $\approx 2.7 \mu \mathrm{m}$ and $3.7 \mu \mathrm{m}$.

We have also included in Fig.13 a scenario where only the propagating part of the plasmonic mode $\omega_{+}(k)$ is populated at a temperature different from the rest of the system. It contributes a free energy

$$
\begin{aligned}
\vartheta_{+}^{\mathrm{pr}}(\lambda, \tau)= & -\aleph(\lambda \tau) \int_{-z_{+}}^{0} \ln \left[1-e^{-\frac{g a}{\lambda \tau}}\right] \mathrm{d} z \\
& -2 \aleph(\lambda \tau)^{3}\left[\mathcal{L}\left(\frac{g_{+}\left(-z_{+}\right)}{\lambda \tau}\right)-\mathcal{L}\left(\frac{g_{+}(0)}{\lambda \tau}\right)\right]
\end{aligned}
$$

where $g_{+}\left(-z_{+}\right)=\sqrt{z_{+}}$as noted before, and

$$
g_{+}(0)=2 \pi \lambda \sqrt{\frac{1}{1+\pi \lambda}}
$$

gives the (dimensionless) wavevector for which the dispersion relation $\omega_{+}(k)$ crosses the light cone. This leads to a nonequilibrium free energy

$$
\tilde{\varphi}_{\text {Lif }}^{\text {neq }}\left(\lambda, \tau_{\text {pr }}, \tau\right)=\varphi_{\text {Lif }}(\lambda, \tau)+\vartheta_{+}^{\text {pr }}\left(\lambda, \tau_{\text {pr }}\right)-\vartheta_{+}^{\text {pr }}(\lambda, \tau),
$$

where $\tau_{\text {pr }}$ is the temperature of the propagating plasmons and $\tau$ is the temperature of all other modes. The corresponding pressure [curve c) in Fig.13] increases with respect to the previous non-equilibrium scenario by approximately a factor of 2 , and repulsion sets in at a somewhat shorter distance. This is because (i) the - otherwise attractive - mode $\omega_{-}(k)$ is less excited and (ii) the propagating branch of $\omega_{+}(k)$ dominates the interaction at these distances and excites the electron plasma on both plates rather than a single one.
The selective excitation of surface plasmon modes is a wellstudied problem (Ref. [26] and references therein). Most of the setups have to cope with the fact that the corresponding electromagnetic field is evanescent and, therefore, cannot be excited directly by laser photons incident from free space. Corrugated surfaces are of some help here [2], and indeed they can convert thermally excited plasmons into far-field radiation [27]. A recently developed four-wave mixing scheme permits to excite surface plasmons even on flat surfaces [26]. The non-equilibrium situation involving only propagating modes [Eq. 411] may be simpler to realize experimentally since these modes couple to free-space light fields and can in principle be excited by shining a laser [28] from the side onto the gap between the mirrors. (See Ref. [29] for a related discussion.)

\section{DISCUSSION AND CONCLUSION}

We have calculated the contribution to the thermal Casimir effect due to surface plasmons, which are hybrid field-matter eigenmodes of metallic surfaces. The expression we found for the free energy of interaction is valid at any distance and temperature, and we have derived its asymptotics at small, intermediate, and large distances. Thermal effects become significant when the distance is larger than the thermal wavelength $\lambda_{T}$, similar to perfectly conducting plates, and below $\lambda_{T}$ for non-equilibrium configurations. The other length scale of the system (plasma wavelength) determines the detailed behavior of the free energy.

We have found that at short distances and temperatures the thermal correction is small, and that the plasmonic Casimir interaction changes sign with distance, leading to a repulsive regime, as has been known from zero temperature [11, 12]. This goes hand in hand with a change of sign of the plasmonic Casimir entropy. In the short-distance regime, we found that the complete Casimir interaction between metallic plates (described by the plasma dielectric function) is completely dominated by the surface plasmon contribution. The asymptotic scaling laws explain why $T=0$ is a good approximation in most experimentally relevant situations (intermediate distance regime, low temperature). In this regime the known result for the complete plasma model is recovered in a simple way by adding propagative photonic modes.

Things are different at high temperatures and large distances. Here it was shown that the plasmonic Casimir interaction is determined by a branch of the surface plasmon dispersion relation corresponding to propagating modes, resulting in a large repulsive contribution that is enhanced by the temperature. This effect is probably one of the best illustrations of Casimir repulsion that arises from the radiation pressure of a standing wave mode. The pressure is repulsive, because the travelling photons are bouncing off the cavity walls, while the reference mode, a single-interface plasmon, has an evanescent field with zero radiation pressure.

The balance between plasmonic and photonic modes was emphasized by considering two configurations out of global thermal equilibrium where plasmonic modes are selectively excited to a higher temperature. These configurations show 
a crossover to a total Casimir force that becomes repulsive at plate distances $L \approx 20 \lambda_{\mathrm{p}} \ldots 25 \lambda_{\mathrm{p}}$ (a few microns for gold). This can be understood qualitatively in terms of radiation pressure due to the propagating branch of the plasmonic mode. We emphasize that this happens at distances shorter than the thermal wavelength where the Casimir pressure is stronger.

In conclusion, it seems in principle possible to tune the sign of the Casimir force by the selective excitation of the surface plasmons. Still, future research must address experimentally relevant questions for such a scheme, e.g. how to avoid exciting photonic modes just above the plasmonic one and how to populate plasmonic modes over a wide angular range.

Acknowledgments. We would like to thank H. T. Dinani and S. Slama for discussions and help with some calculations. We benefited from exchanging ideas within the Research Network "Casimir" of the European Science Foundation (ESF). FI acknowledges partial financial support by the Humboldt foundation and LANL. HH and $\mathrm{CH}$ acknowledge funding by the German-Israeli Foundation for Development and Research (GIF).

\section{Appendix A: Full Casimir entropy at low temperatures}

The dimensionless correction factor for the Casimir entropy of the plasma model can be written as the following integral over (scaled) real frequencies

$$
\sigma_{\text {Lif }}(\lambda, \tau)=-\frac{4}{\pi \zeta(3)} \int_{0}^{\infty} \frac{x \mathrm{~d} x}{\sinh ^{2} x} \operatorname{Im} \sum_{p} M_{p}(2 x \lambda \tau)
$$

where $p=$ TE, TM indicates again the polarization and

$$
\begin{aligned}
M_{p}(\Omega) & =\int_{0}^{\infty} \mathrm{d} \kappa \kappa \ln \left[1-r_{p}^{2}(\Omega, \kappa) e^{-2 \kappa}\right] \\
& +\int_{0}^{\Omega} \mathrm{d} y y \ln \left[1-r_{p}^{2}(\Omega,-\mathrm{i} y) e^{2 \mathrm{i} y}\right]
\end{aligned}
$$

The first (second) integral in Eq. A2 corresponds to the evanescent wave (propagating wave) sector, respectively. For $p=\mathrm{TE}$, the argument of the logarithm is always positive in the first integral, hence its imaginary part vanishes. This does not happen for $p=\mathrm{TM}$ where the first integral gives the contribution of surface plasmons (evanescent branch) which has been evaluated in this paper. As mentioned in Sec. $\mathrm{V}$, we are interested here in the propagating contribution only.

The function $x / \sinh ^{2} x$ significantly differs from zero only for $x \lesssim 1$. In the limit $\lambda \tau \ll 1$, we can therefore expand the integrands in $M_{p}(\Omega)$ for small $y$ and $\Omega$ since $y \leq \Omega \ll 1$. This yields

$$
\begin{aligned}
& \operatorname{Im} M_{\mathrm{TE}}^{\mathrm{ph}}(\Omega) \approx-\frac{\pi}{4} \Omega^{2}+\frac{\Omega^{3}}{3 \pi \lambda}(1+\pi \lambda) \\
& \operatorname{Im} M_{\mathrm{TM}}^{\mathrm{ph}}(\Omega) \approx-\frac{\pi}{4} \Omega^{2}+\frac{\Omega^{3}}{3 \pi \lambda}(3+\pi \lambda)
\end{aligned}
$$

Performing the $x$-integral in Eq. A1,

$$
\sigma_{\mathrm{ph}}(\lambda, \tau) \underset{\tau \ll 1}{\stackrel{\lambda \tau \ll 1}{\longrightarrow}} \sigma_{\mathrm{C}}(\lambda, \tau)\left[1-\frac{8 \pi^{2} \tau}{45 \zeta(3)} \frac{2+\pi \lambda}{3}\right]
$$

Note, however, that this result contains the propagating branch of the plasmonic mode $\omega_{+}(k)$ whose free energy is given by Eq. 39. Reviewing the analysis from Secs. III A IIIB, it is easy to see that in the limit considered here, the polylogarithmic term dominates in Eq. 39 and becomes $\mathcal{L}\left(\sqrt{z_{+}} / \lambda \tau\right) \approx \zeta(3)$. Subtracting this contribution from A5, we find the entropy of the propagating photonic modes given in Eq. (34).
[1] E. N. Economou. Phys. Rev. 182, 539 (1969).

[2] H. Raether, Surface Plasmons on Smooth and Rough Surfaces and on Gratings, volume 111 of Springer Tracts in Modern Physics (Springer, Berlin 1988).

[3] N. V. Kampen, B. Nijboer, and K. Schram, Phys. Lett. A 26, 307 (1968); K. Schram, Phys. Lett. A 43, 282 (1973).

[4] E. Gerlach, Phys. Rev. B 4, 393 (1971).

[5] M. L. Brongersma and P. G. Kik, eds., Surface Plasmon Nanophotonics, Springer Series in Optical Sciences (Springer, Berlin Heidelberg New York 2007).

[6] S. A. Maier, Plasmonics: Fundamentals and Applications (Springer, Berlin Heidelberg New York 2007).

[7] F. J. Garcia-Vidal, L. Martin-Moreno, T. W. Ebbesen, and L. Kuipers, Rev. Mod. Phys. 82, 729 (2010).

[8] E. Altewischer, M. van Exter, and J. Woerdman. Nature, 418,
304 (2002).

[9] S. Fasel, F. Robin, E. Moreno, D. Erni, N. Gisin, and H. Zbinden. Phys. Rev. Lett., 94, 110501 (2005).

[10] F. Intravaia, PhD thesis, Univ. Paris VI, 2005.

[11] F. Intravaia and A. Lambrecht, Phys. Rev. Lett. 94, 110404 (2005).

[12] F. Intravaia, C. Henkel, and A. Lambrecht, Phys. Rev. A 76, 033820 (2007).

[13] H. Casimir, Proc. kon. Ned. Ak. Wet. 51, 793 (1948).

[14] M. Bordag, J. Phys. A 39, 6173 (2006).

[15] Z. Lenac, Phys. Rev. Lett. 96, 218901 (2006).

[16] A. Lambrecht and I. G. Pirozhenko, Phys. Rev. A 78, 062102 (2008).

[17] J. Feinberg, A. Mann, and M. Revzen, Ann. Phys. (N.Y.) 288, 103 (2001). 
[18] V. Bezerra, G. Klimchitskaya, and V. Mostepanenko, Phys. Rev. A 65, 052113 (2002).

[19] E. Lifshitz, Sov. Phys. JETP 2, 73 (1956) [J. Exper. Theoret. Phys. USSR 29, 94 (1955)].

[20] V. B. Bezerra, G. L. Klimchitskaya, and V. M. Mostepanenko, Phys. Rev. A 66, 062112 (2002).

[21] V. B. Bezerra, G. L. Klimchitskaya, V. M. Mostepanenko, and C. Romero, Phys. Rev. A 69, 022119 (2004).

[22] I. Brevik, S. A. Ellingsen, and K. A. Milton, New J. Phys. 8, 236 (2006).
[23] I. A. Dorofeyev, J. Phys. A 31, 4369 (1998).

[24] M. Antezza, L. P. Pitaevskii, S. Stringari, and V. B. Svetovoy, Phys. Rev. A 77, 022901 (2008).

[25] L. P. Pitaevskii, J. Phys. A 39, 6665 (2005).

[26] J. Renger, R. Quidant, N. van Hulst, S. Palomba and L. Novotny, Phys. Rev. Lett. 103, 266802 (2009).

[27] J.-J. Greffet, R. Carminati, K. Joulain, J.-P. Mulet, S. Mainguy, and Y. Chen, Nature 416, 61 (2002).

[28] M. L. Povinelli et al., Opt. Lett. 30, 3042 (2005).

[29] Y. B. Ovchinnikov, Opt. Commun. 182, 35 (2000). 